\title{
Cross-Departmental Access to Relevant Clinical Information for Early Rehabilitation Using a Web-Based Medical Multimedia Document Server
}

\author{
M Eichelberg ${ }^{1}$, K Kronberg ${ }^{2}$, D Heidkamp ${ }^{3}$, M Gründler ${ }^{1}$, \\ $\mathrm{O} \mathrm{Nee}^{1}, \mathrm{H}$ Spekker ${ }^{1}$ \\ ${ }^{1}$ OFFIS eV, Oldenburg, Germany \\ ${ }^{2}$ Klinikum Oldenburg, Department of Cardiology, Oldenburg, Germany \\ ${ }^{3}$ Rehabilitations-Zentrum Oldenburg, Oldenburg, Germany
}

\begin{abstract}
The "Mobile Multimedia Medical Information System" $\left(M^{3} I S\right)$ project develops a system that enables the exchange of clinical documents, medical images, films and laboratory results both within a hospital and between healthcare institutions. The system accepts documents through DICOM and HL7 interfaces and provides a webbased client interface that allows for a quick and easy access to the system through a web browser. In order to comply with data protection requirements, the system implements a powerful access control scheme. The system ensures that most clinically relevant document are available to early rehabilitation in a timely manner, before the final written discharge report becomes available.
\end{abstract}

\section{Introduction}

The digital acquisition and management of clinical data is a standard today in most clinical departments and private practices. However, most often the patient treatment does not take place in a single hospital department or a single practice only. The chain of care usually involves multiple institutions such as hospital, rehabilitation center, specialist practice and general practitioner, thus creating a need for an exchange of all relevant information between the actors involved. Ideally, the information required for patient care should be available at any time at the place where needed. However, in the absence of a comprehensive electronic healthcare record, access to clinical information is not always easy. We examined the preliminary reports transmitted from cardiology and cardiac surgery to the early rehabilitation along with the patient in the Integrated Heart Center in Oldenburg, Germany, which includes the clinics of cardiology, cardiac surgery and cardiac rehabilitation on a single campus. We found that clinical history, diagnoses, medication or laboratory data were frequently incomplete in the hand-written reports. The full discharge letters, however, were available too late for early rehabilitation. For this department alone, the time required to acquire missing information was estimated at 330 hours annually.

\section{Methods}

An integrated system for an interchange of medical documents in the care chain was developed in the framework of the $\mathrm{M}^{3} \mathrm{IS}$ project (Mobile Multimedia Medical Information System) [1]. The M ${ }^{3}$ IS system comprises a document and communication server that mediates between the various clinical systems such as the Cardiology Information System (CIS), the laboratory system, the departmental system for cardiac surgery, and the Hospital Information System (HIS). The system accepts documents in various formats, including diagnostic reports, DICOM (Digital Imaging and Communications in Medicine) images and films, laboratory data and discharge letters, through standard DICOM and HL7 (Health Level Seven) interfaces, but also through proprietary interfaces where necessary.

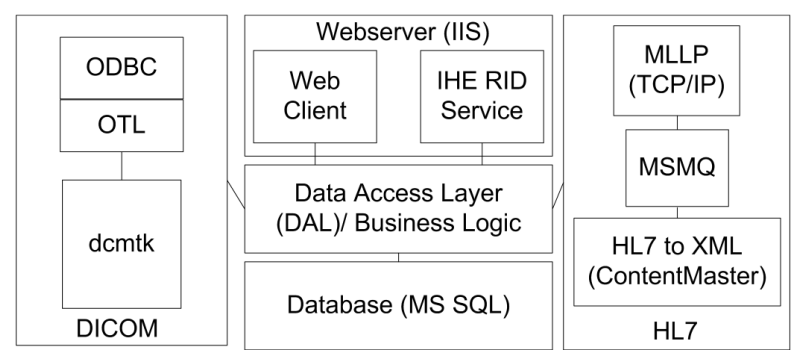

Figure 1: $\mathrm{M}^{3}$ IS system modules

The system uses two servers, one within the hospital's network (i. e., behind the institution's firewall) for internal communication within the hospital, and one that is accessible from the outside world through internet or dial-in connections and used exclusively for communication with external parties (such as private practices). Fig 1 shows the system modules of which the so-called "internal server" is comprised. The central system database is encapsulated through an object- 
relational "data access layer" on top of which various services operate. There are two web-based access services, one providing a web client user interface that can be used to interact with the system directly through a web browser, and a Web Service interface named "Retrieve Information for Display" [2] based on a specification of the Integrating the Healthcare Enterprise (IHE) initiative [3] which enables read-only access from other clinical systems to the documents managed by the M ${ }^{3}$ IS system. Both web-based services are delivered through a standard Microsoft Internet Information Server. The DICOM interface is based on the Open Source OFFIS DICOM Toolkit (DCMTK) and accesses the database through a library named "Oracle Template Library" (OTL) [4] that provides a common application programming interface for database access, which in this case takes place through ODBC (Open Database Connectivity) [5]. The HL7 interface is based on the HL7 "minimal lower layer protocol" (MLLP), the Microsoft Message Queue (MSMQ) and an HL7 toolkit named Itemfield ContentMaster [6]. The external server, which is not shown in Fig. 1, actually comprises a subset of the components of the internal server and stores in encrypted form only those documents that have been configured for access by certain external users.

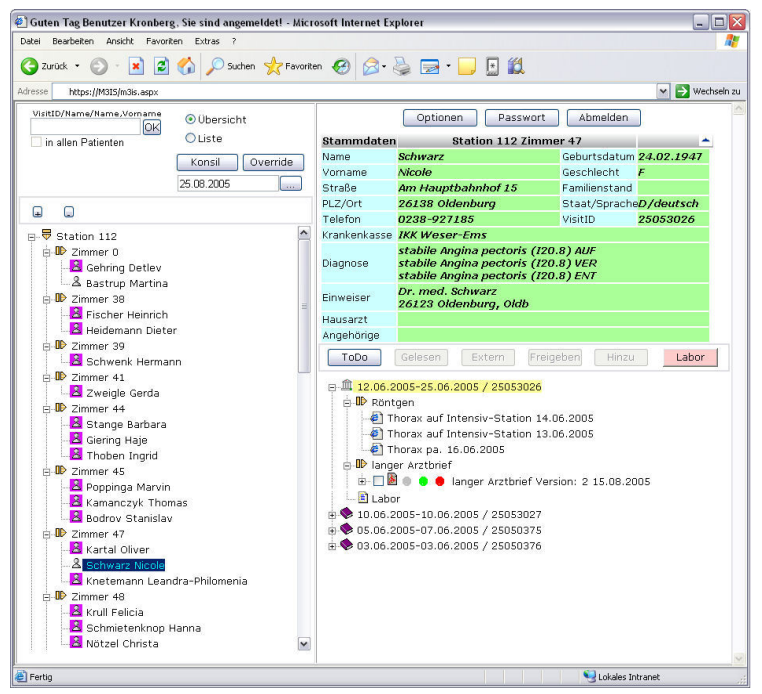

Figure 2: $\mathrm{M}^{3} \mathrm{IS}$ web client user interface

Fig. 2 shows the graphical user interface of the Web Client that is currently used by medical staff to access clinical documents available through the system. The lefthand side of the screen shows a list of wards, rooms and patients to which the current user has access. The righthand side shows the demographics and list of diagnoses of the currently selected patient along with the list of documents available for the patient, ordered by visit and type of document. All names shown in this and the following figures have been replaced by random data. It should be noted that most interactions with the system only require few mouse clicks and can easily be performed with mobile devices that have no keyboard, such as Tablet PCs or Web Pads and can be used to access the system, e. g. during the ward round.

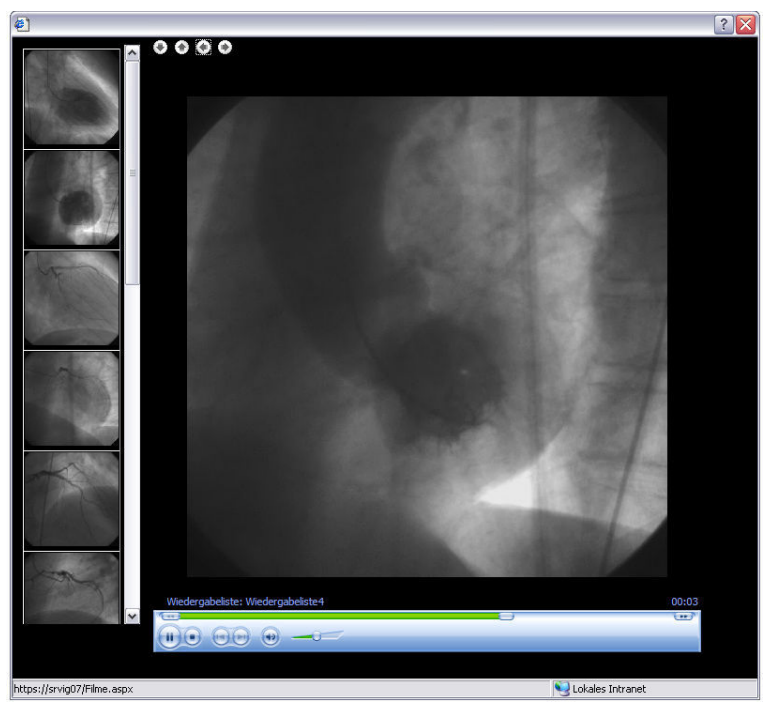

Figure 3: $\mathrm{M}^{3} \mathrm{IS}$ web viewer for cath-lab films

Fig. 3 shows the viewer used within the web client to visualize heart catheterization and echocardiography films. In order to save bandwidth, an issue of importance both for external users and mobile devices connected through wireless networks, the film sequences are not transmitted in their original DICOM format, but in the MPEG4 compressed format defined by the Motion Picture Expert Group. This means that image quality is not suitable for the primary diagnosis, which is done in the catheterization laboratory. However, in our experience $[7,8]$ the image quality is quite appropriate for review purposes under which they are used with our system.

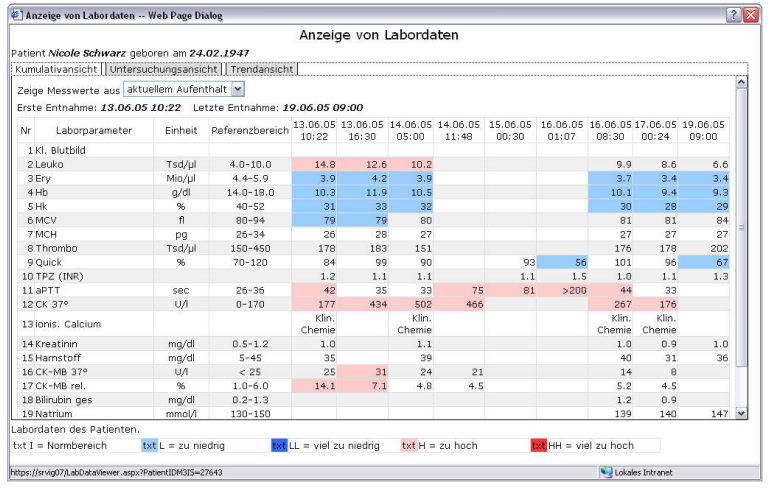

Figure 4: $\mathrm{M}^{3} \mathrm{IS}$ web viewer for laboratory results 
Fig. 4 shows the web viewer for laboratory data, which is the only type of data that is not document based since individual results can be delivered by the laboratory system through HL7 messages. The viewer allows to visualize all laboratory results for the current or multiple visits of the patient, including a trend view for individual parameters and the cumulative view shown in Fig. 4 in which abnormal results are color coded.

A critical issue for a system that manages the exchange of clinical documents between departments and even between different healthcare enterprises is the management of access rights. Data protection regulations such as the EU directive 95/46/EC [9] require that patient related documents must be available only to authorized medical staff, which is typically the staff directly involved in the provision of care to the patient. In addition, physicians attach great importance to a quality assurance process ensuring that only validated diagnostic reports or discharge letters are made available beyond department boundaries, both for medico-legal reasons and for considerations of patient safety.

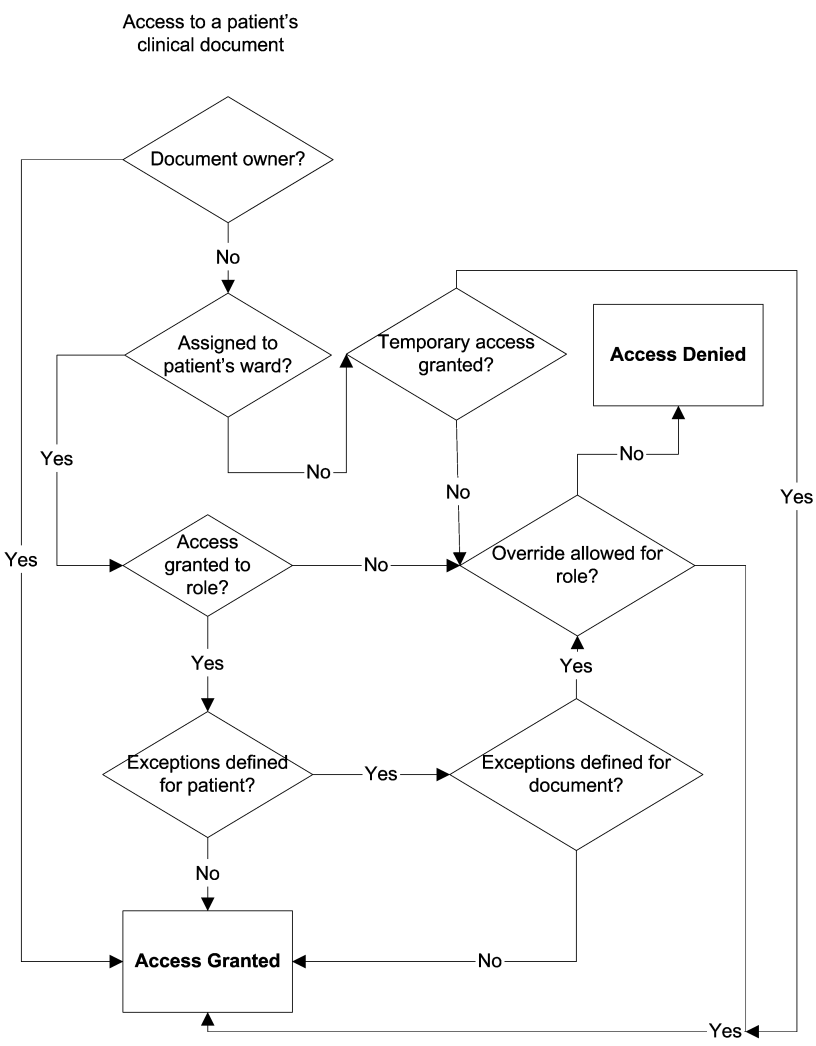

Figure 5: Organization based access control in $\mathrm{M}^{3} \mathrm{IS}$

The Mㄴ $\mathrm{M}^{3} \mathrm{~S}$ system addresses this issue by automatically tracking patient admissions, discharges and interdepartmental transferals. Based on that data, it determines where, and to whom specific information should be made available, respecting any restrictions that may have been defined by the document author. Fig. 5 shows the organization based access control system that decides whether or not a specific user is entitled to access a certain document. In general, a user has always access to all documents he or she has authored. In addition, access is granted if the patient is or was assigned to one of the user's wards within the last couple of days, the document is available to the role under which the user operates (e. g., ward physician) and no exceptions have been defined for either the patient (e. g., VIP patient with access restricted to certain users) or the document (e. g. classified document accessible only to the original author). Authorized users can grant a temporary access right to other users for the purpose of medical consultations. In all other cases, access is denied. Finally, there is a concept called "emergency override" that is available to certain users (roles). If for some reason an important document that is available in the system is inaccessible but needed for the immediate treatment process, the user can force access to the document by enabling the emergency override mode. In this mode, however, all activities are reported automatically to the hospital's data protection officer who may then initiate an a-posteriori enquiry in which the system user is requested to explain the need for access to the document. All transmission from the server system to the client takes place in encrypted form so that confidentiality is guaranteed even over wireless connections or in long-distance campus networks. Finally, the quality assurance process mentioned above is implemented in the system for reports and discharge letters that may be revised and validated by users of certain roles (senior physician, head of department) and only become available to other departments once a certain type of validation is registered.

\section{Results}

The system significantly improves access to clinical information by seamlessly making available many relevant information sources before the final discharge letter is compiled. Currently all films, images and reports from the catheterization and the echocardiography laboratory are available in the system along with the heart surgery, gastroenterology and radiology reports, laboratory results and all historical records of the patient which are available as an electronic record based on scanned paper documents. All of these documents are submitted automatically by the various clinical systems in the hospital. Discharge reports from cardiology and heart surgery are available when uploaded to the system - short reports are created by the cardiology information system, the more comprehensive ones still undergo conventional 
voice dictation and transcription. Nevertheless, the majority of information sources relevant to the early rehabilitation is now available in a timely manner. Remaining open issues are the rapid access to ECG data due to the lack of digital interfaces in most ECG devices and the absence of an ECG management system in the heart center. The nursing documentation is currently still in paper form, which also means that the most recent medication of the patient is not available to the system in digital form.

The client-side configuration of the system has proven to be rather unproblematic since only a modern web browser such as the Microsoft Internet Explorer 6 or Mozilla/Firefox is needed, along with TCP/IP connectivity to the internal $\mathrm{M}^{3} \mathrm{IS}$ server. We have also successfully used mobile clients operating under an embedded operating system (Windows CE).

\section{Discussion and conclusions}

Given the experiences from the ongoing $\mathrm{M}^{3} \mathrm{IS}$ project, the authors believe that the problem most difficult to tackle is the appropriate mapping of the clinical workflow with the system. Only a near real-time clinical documentation in digital form guarantees an early availability of information along the care chain. Such an immediate and structured clinical documentation may in the end result in a benefit for the patient but is not necessarily perceived as an improvement by the medical staff if changes in the work process are involved, given the high workload under which hospitals are operating today. The problem is that often benefits are realized "somewhere else", but the costs of workflow optimizations (e.g., increased work load for certain members of the team, learning curve for new organizational procedures) occur locally. Only if the users perceive workflow changes as something that also improves their own working environment by saving their time, user acceptance can be expected. Examples for such improvements are an easier availability of information that physicians need for their daily work as well as "intelligent" services offered by the system such as a semi-automatic report creation and the maintenance of worklists.

The description of the access control scheme for $\mathrm{M}^{3} \mathrm{IS}$, which is actually only a small part of the comprehensive set of technical security measures implemented for the system, shows the significant complexity of developing a system that supports clinical workflows within and across department and institution boundaries and that also complies with existing European data protection laws. The authors believe that information technology security needs to be an integral part of a system's design. It is illusory to believe that appropriate security measures can be implemented as an add-on to a system that has only been designed for administrative and clinical functional requirements, but without consideration to data protection requirements in particular for inter-institutional communication. A system like $\mathrm{M}^{3} \mathrm{IS}$ will always find itself in an area of conflict between the patient's privacy rights, patient-doctor confidentiality, and the users' desire to access the data they need for their work without too many restrictions. Practical experience shows that security measures that are not accepted by the users will be circumvented, so system designers need to find a way of implementing security in a way that is as transparent to the user as possible. Biometrics or wireless identification tags may be a viable option for this purpose.

\section{Acknowledgements}

The M3S Project is supported by the State of Lower Saxony in the framework of the Multimedia Initiative Lower Saxony (Multimedia-Initiative Niedersachsen).

\section{References}

[1] M3S Project Homepage. http://www.m3is.de/

[2] Integrating the Healthcare Enterprise, IHE IT Infrastructure Technical Framework Volume 1, http://www.ihe.net/ tf/ihe_iti_tf_1.1_vol1_FT.pdf

[3] Integrating the Healthcare Enterprise. http://www.ihe.net/

[4] Sergei Kuchin, The Oracle, ODBC and DB2-CLI Template Library $(O T L)$, http://otl.sourceforge.net/

[5] Microsoft Corporation, Microsoft ODBC Application Programmer's Guide, 1992

[6] Itemfield, Itemfield ContentMaster 3.2 Technical Overview, http://www.itemfield.com/news/downloads.asp?file= /pdf/CM_TechOverview.pdf

[7] Kronberg K, Claus M, Riesmeier J, Eichelberg M, Reil GH: One year experience with a Cardiology PACS using $M P E G-4$ for video compression, in: Proceedings of the 20th EuroPACS annual meeting, Oulu University Press, p. 219 (2002)

[8] Wolfgang Vocke, Anwendung von Videokompressionsalgorithmen zur Datenreduktion koronarangiographischer Untersuchungen, MD Thesis, Medizinische Hochschule Hannover (2002) (in German language)

[9] Directive 95/46/EC of the European Parliament and of the Council of 24 October 1995 on the protection of individuals with regard to the processing of personal data and on the free movement of such data, OJ L, 23 November 1995

Address for correspondence

Dr. Marco Eichelberg

OFFIS e.V., Escherweg 2, D-26121 Oldenburg, Germany

eichelberg@offis.de 